\title{
Post lumbar puncture discitis and vertebral collapse
}

\author{
H.S. Bhatoe, H.S. Gill, N. Kumar and S. Biswas \\ Departments of Neurosurgery and Neurology, Command Hospital(SC), Pune 411040; Maharashtra, India
}

\begin{abstract}
Summary: Discitis and vertebral collapse are rare but serious complications of lumbar puncture. Clinical profile, management and course of post lumbar puncture discitis in five patients is presented. Such a complication should be kept in mind whenever any invasive procedure is carried out on the spine.
\end{abstract}

\section{Introduction}

In modern day hospital practice, there are a number of indications that warrant access to the spinal subarachnoid space. Barring venepuncture, lumbar puncture (LP) is probably the most common invasive procedure undertaken. The side effects of LP are few: mild headache, which responds to analgesics and increased fluid intake, is the commonest. Serious complications include meningitis, and rarely discitis and vertebral collapse. ${ }^{\prime}$ We present five patients with post-LP discitis, one of whom had vertebral collapse. The relevant literature is reviewed.

\section{Clinical material}

There were five patients with post-LP discitis seen in our hospital over a period of 7 years. Two of these patients developed discitis after LP in our hospital and three were referred from other hospitals. All patients were males in their third to fifth decades. The indications of LP, problems encountered during puncture, incubation period and radiological findings are given in Table I. Clinical profile of all five patients was similar: severe backache, paraspinal muscle spasm, inability to raise leg straight, no neurological signs and elevated erythrocyte sedimentation rate. All five were managed with bed rest, antibiotics (cloxacillin/ampicillin and gentamicin) for 6 weeks. They were allowed out of bed after 6 weeks and were followed up as outpatients. They all became pain free between 6 and 12 months. Spine radiographs after 12 months showed fusion across the infected disc space with formation of lateral spurs.

\section{Discussion}

Damage to an intervertebral disc is always a possibility during LP. It is likely that a number of

Correspondence: H.S. Bhatoe, M.S., M.Ch.

Accepted: 3 May 1994 mild backache cases after LP may have a spontaneously resolving condition. ${ }^{2}$ The needle pierces the annulus, producing varying sized haematoma, especially if repeated attempts are made, eventually predisposing it to infection. Even though scrupulous sterile technique is employed in most lumbar punctures, occasionally organisms will be introduced into the above locus minoris resistentiae. Blankenstein et al. ${ }^{4}$ described in detail how the intervertebral disc is initially infected and bacteria are introduced into the disc space at the time of intervention. A disc space infection ensues and the organisms are almost always confined to the dis space where the disc elements undergo sequestras tion, liquefaction and eventual resorption, wit interspace collapse. Confinement of the infection to the interspace is probably due to a combination of cartilaginous plate barrier and the rich vascularity of the vertebral body, which prevents its sequestration and destruction; a vertebral osteomyelitis may ensue when the cartilaginous plate is damaged. Such a situation may arise after multiple punctures have been made in two adjacent spaces.

Clinically, post-LP discitis is characterized by a typical presentation. About two weeks after LP, the patient develops severe low back pain. There is marked paravertebral muscle spasm, and, occasionally, fever. Phýsical examination is remarkable for one thing: the paucity of neurological signs in the face of extreme pain suffered by the patient. Straight leg raising may be markedly reduced or cannot be tested.

Laboratory findings in these patients tend to be within normal limits, with the exception of raised ESR. Radiological changes may appear within 2-6 weeks. There is haziness or blurring of epiphyseal plates adjacent to the disc space. Destruction of the disc and that of the vertebral body can be seen clearly by computed tomography (CT) (Figures 1 and 2). The most effective early demonstration of suspected spondylodiscitis before any changes are visible radiographically ${ }^{6}$ is by magnetic resonance imaging. 
Table I Discitis following lumbar puncture in five patients

\begin{tabular}{llllll}
\hline $\begin{array}{l}\text { Patient } \\
\text { no. }\end{array}$ & Indication for LP & Remarks & Incubation period & Investigation & Radiological findings \\
\hline 1 & Spinal analgesia & $\begin{array}{l}\text { Repeated punctures } \\
\text { at two spaces }\end{array}$ & 2 weeks & X-ray, CT & $\begin{array}{l}\text { Discitis L2/L3, L3/ } \\
\text { L4 with collapse L3 }\end{array}$ \\
2 & Spinal analgesia & Repeated punctures & 6 weeks & X-ray & Discitis L3/L4 \\
3 & Spinal analgesia & Repeated punctures & 4 weeks & X-ray & Discitis L3/L4 \\
4 & Diagnostic & Single puncture & 3 weeks & X-ray, CT & Discitis L4/L5 \\
5 & Cranial CSF fistula & $\begin{array}{l}\text { Twice daily punc- } \\
\text { ture for 5 days }\end{array}$ & 3 months & X-ray, CT & Discitis L3/L4 \\
\hline
\end{tabular}

LP = lumbar puncture; $C S F=$ cerebrospinal fluid; $C T=$ computed tomography.

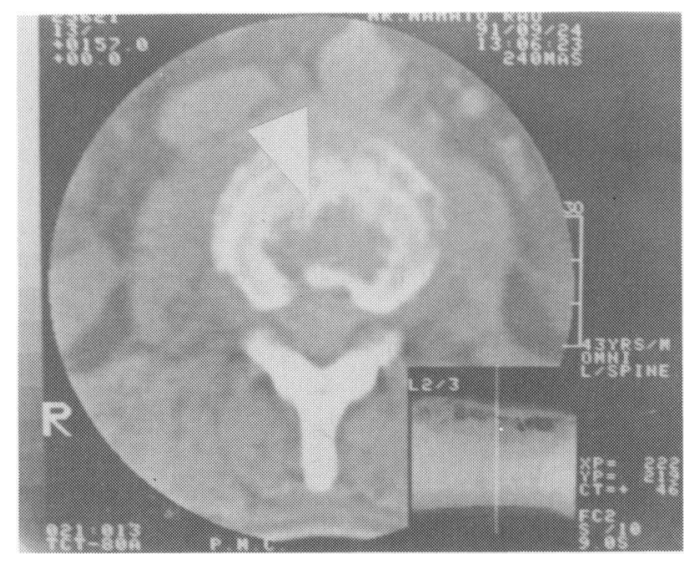

Figure 1 CT showing discitis L2/L3 (arrow).

There is convincing evidence that the changes of discitis are the result of an infective process. ${ }^{7}$ Only a small number of bacteria are necessary to produce this complication and within weeks of it being established, the causal organisms cannot be isolated. ${ }^{7}$ In both the experimental animal and material obtained at autopsy, the histological picture is that of a chronic inflammatory process. ${ }^{8}$

The course of post-LP discitis appears to be similar to that of post-discectomy disc space infection. As time progresses, the disc space becomes markedly reduced and vertebral plates become indistinct. The space may disappear and healing begins with new bone formation in the form of sclerotic vertebral margins and spurs. In most

\section{References}

1. Weatherly, C.R., Jaffray, D., Mehta, M. \& O'Brien, J.P. Vertebral collapse following spinal anaesthesia. Spine 1991, 16: 592-593.

2. McCall, I.W. Radiologic assessment of back pain. Semin Orthop 1986, 1: 71-85.

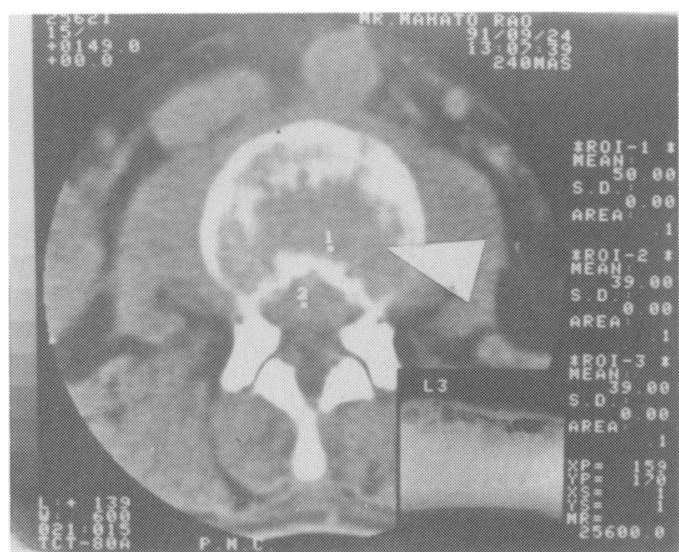

Figure 2 CT showing L3 vertebral body destruction (arrow).

patients, healing and new bone formation leads to bony fusion. The process from active destruction to fusion takes anywhere from 6 months to 2 years.

Management of post-LP discitis includes bed rest, analgesics and antibiotics for a period of 6 weeks. Generally a combination of semisynthetic penicillin and aminoglycoside is preferred. Our patients were relatively pain free and ambulant after 6 weeks. We do not recommend use of a rigid spinal brace. The sequence reported in these patients with one proceeding to bony destruction is rare; nonetheless such a complication should be borne in mind whenever any invasive procedure is carried out on the spine or its contents.

3. Pilgaard, S. Discitis (closed space infection) following removal of intervertebral disc. J Bone Joint Surg 1969, 51A: 713-716. 
4. Blankenstein, A., Rubinstein, E., Ezra, E., Lokiec, F., Caspi, I. \& Horoszowski, H. Disc space infection and vertebral osteomyelitis as a complication of percutaneous lateral discectomy. Clin Orthop 1987, 225: 234-237.

5. Scherbal, A.L. \& Gardner, W.J. Infections involving the intervertebral discs: diagnosis and management. JAMA 1960, 174: $370-374$.

6. Enzmann, D.R., De La Paz, R.L. \& Bubin, J.B. In: Magnetic Resonance Imaging of the Spine. C.V. Mosby Co., St Louis, 1990, pp. 262-264.
7. Fraser, R.D., Osti, O.L. \& Vernon Roberts, B. Discitis following discography - An experimental study. Orthop Trans 1985, 9: 530-531.

8. Fraser, R.D., Osti, O.L. \& Vernon Roberts, B. Discitis following discography - Incidence and pathological findings. Orthop Trans 1985, 9: 532. 